\title{
Diacylglycerol Lipase Is Not Involved in Depolarization-Induced Suppression of Inhibition at Unitary Inhibitory Connections in Mouse Hippocampus
}

\author{
Rogier Min, ${ }^{1}$ Guilherme Testa-Silva, ${ }^{1}$ Tim S. Heistek, ${ }^{1}$ Cathrin B. Canto, ${ }^{2}$ Johannes C. Lodder, ${ }^{1}$ Tiziana Bisogno, ${ }^{3}$ \\ Vincenzo Di Marzo, ${ }^{3}$ Arjen B. Brussaard, ${ }^{1}$ Nail Burnashev, ${ }^{1,4}$ and Huibert D. Mansvelder ${ }^{1}$ \\ ${ }^{1}$ Department of Integrative Neurophysiology, Center for Neurogenomics and Cognitive Research, Neuroscience Campus Amsterdam, VU University, 1081 \\ HV Amsterdam, The Netherlands, ${ }^{2}$ Kavli Institute for Systems Neuroscience and Centre for the Biology of Memory, Norwegian University of Science and \\ Technology, NO-7489 Trondheim, Norway, ${ }^{3}$ Endocannabinoid Research Group, Institute of Biomolecular Chemistry, Consiglio Nazionale delle Ricerche, \\ 80078 Puzzuoli (Naples), Italy, and ${ }^{4}$ Department of Anatomy and Neurosciences, VU Medical Center, 1081 BT Amsterdam, The Netherlands
}

Endocannabinoids control hippocampal inhibitory synaptic transmission through activation of presynaptic $\mathrm{CB}_{1}$ receptors. During depolarization-induced suppression of inhibition (DSI), endocannabinoids are synthesized upon postsynaptic depolarization. The endocannabinoid 2-arachidonoylglycerol (2-AG) may mediate hippocampal DSI. Currently, the best studied pathway for biosynthesis of 2-AG involves the enzyme diacylglycerol lipase (DAGL). However, whether DAGL is necessary for hippocampal DSI is controversial and was not systematically addressed. Here, we investigate DSI at unitary connections between $\mathrm{CB}_{1}$ receptor-containing interneurons and pyramidal neurons in CA1. We found that the novel DAGL inhibitor OMDM-188, as well as the established inhibitor RHC-80267, did not affect DSI. As reported previously, effects of the DAGL inhibitor tetrahydrolipstatin depended on the application method: postsynaptic intracellular application left DSI intact, while incubation blocked DSI. We show that all DAGL inhibitors tested block slow self-inhibition in neocortical interneurons, which involves DAGL. We conclude that DAGL is not involved in DSI at unitary connections in hippocampus.

\section{Introduction}

Endocannabinoids mediate both short- and long-term plasticity at many synapses in the brain. The first form of synaptic plasticity in which endocannabinoids were shown to be involved is hippocampal depolarization-induced suppression of inhibition (DSI) (Wilson and Nicoll, 2001). Hippocampal DSI occurs at a subset of inhibitory synapses onto pyramidal neurons coming from $\mathrm{CB}_{1}$ receptor $\left(\mathrm{CB}_{1} \mathrm{R}\right)$-containing interneurons. At these synapses, a postsynaptic depolarization leads to calciumdependent synthesis of endocannabinoids, which subsequently travel to the presynaptic inhibitory terminal. Here, endocannabinoids activate the G-protein coupled $\mathrm{CB}_{1} \mathrm{R}$, thereby reducing presynaptic release probability for tens of seconds. The endocannabinoid 2-arachidonoyl glycerol (2-AG) (Sugiura et al., 1995)

\footnotetext{
Received July 27, 2009; revised Dec. 23, 2009; accepted Jan. 4, 2010

The Department of Integrative Neurophysiology is financially supported by NeuroBsik (www.neurobsik.nl), NWO and the VU University board. We thank B. Lutz for providing us with $\mathrm{CB}_{1} \mathrm{R}^{-1-}$ mice, K. Mackie for providing us with the $\mathrm{CB}_{1}$ R antibody, N. Lozovaya and T. Nevian for helpful discussions, and J. Timmerman and T. Lodder for excellent technical support. R.M., A.B.B., N.B., and H.D.M. designed the experiments. R.M., G.T.-S., T.S.H., and J.C.L. performed experiments; C.B.C. performed immunohistochemistry and reconstructions; T.B. and V.D.M. synthesized OMDM-188; R.M. analyzed the data; R.M. and H.D.M. wrote the manuscript; all authors commented on the manuscript.

Correspondence should be addressed to either of the following: Huibert D. Mansvelder, Department of Integrative Neurophysiology, Center for Neurogenomics and Cognitive Research, Neuroscience Campus Amsterdam, VU University, De Boelelaan 1085, Room C-440, 1081 HV Amsterdam, The Netherlands, E-mail: huibert. mansvelder@ cncr.vu.nl; or Nail Burnashev, Institut de Neurobiologie de la Méditerranée, Parc Scientifique de Luminy, 163 Roude de Luminy, BP13, 13273 Marseille, France, E-mail: nail.burnashev@inmed.fr.

DOI:10.1523/JNEUROSCI.BC-3622-09.2010

Copyright $\odot 2010$ the authors $\quad 0270-6474 / 10 / 302710-06 \$ 15.00 / 0$
}

has been proposed to mediate hippocampal DSI, since inhibiting 2-AG degradation increases the duration of DSI (Kim and Alger, 2004; Makara et al., 2005; Hashimotodani et al., 2007). However, $s n$-1-diacylglycerol lipase (DAGL) $\alpha$, the major enzyme catalyzing 2-AG formation in the adult brain (Bisogno et al., 2003) has not been found opposite symmetrical, GABAergic synapses in hippocampus (Katona et al., 2006). Furthermore, studies in which DAGLs have been targeted pharmacologically reported conflicting results. Blocking these enzymes pharmacologically suppresses hippocampal DSI in some studies (Hashimotodani et al., 2007) but not in others (Chevaleyre and Castillo, 2003; Edwards et al., 2006; Szabo et al., 2006). So far, the only study showing pharmacological evidence for DAGL involvement in hippocampal DSI was performed on cultured neurons. This raises the question whether DAGL is involved in hippocampal DSI at intact synapses. Here, by using three chemically different DAGL inhibitors, we have investigated DAGL involvement in hippocampal DSI at unitary connections between $\mathrm{CB}_{1} \mathrm{R}$ containing interneurons and CA1 pyramidal neurons.

\section{Materials and Methods}

Slice preparation. All experiments were approved by the Animal Ethical Committee of the VU University Amsterdam, in accordance with Dutch and European law. For DSI experiments, 14- to 21-d-old wildtype $\mathrm{C} 57 \mathrm{~B} 16$ mice or $\mathrm{CB}_{1} \mathrm{R}^{-1-}$ mice (Marsicano et al., 2002) were decapitated. Transverse hippocampal slices $(300 \mu \mathrm{m})$ were sectioned in ice-cold solution containing ( $\mathrm{mm}): 126 \mathrm{NaCl}, 3 \mathrm{KCl}, 10$ glucose, 26 $\mathrm{NaHCO}_{3}, 1.2 \mathrm{NaH}_{2} \mathrm{PO}_{4}, 1 \mathrm{CaCl}_{2}$, and $3 \mathrm{MgCl}_{2}$ (carboxygenated with $5 \% \mathrm{CO}_{2} / 95 \% \mathrm{O}_{2}$ ). For slow self-inhibition ( $\mathrm{SSI}$ ) experiments, sagittal 


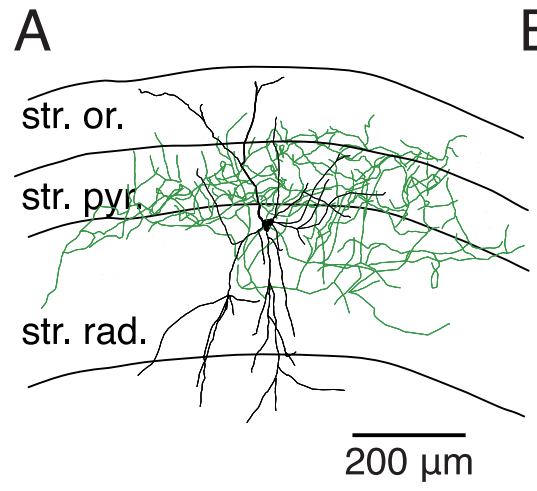

B

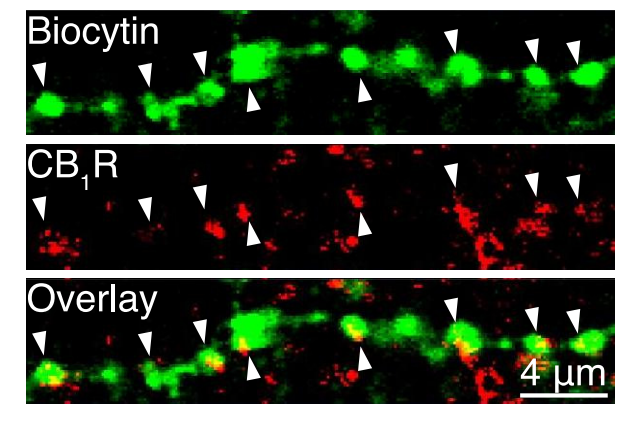

C $\mathrm{CB}_{1} \mathrm{R}$ interneuron
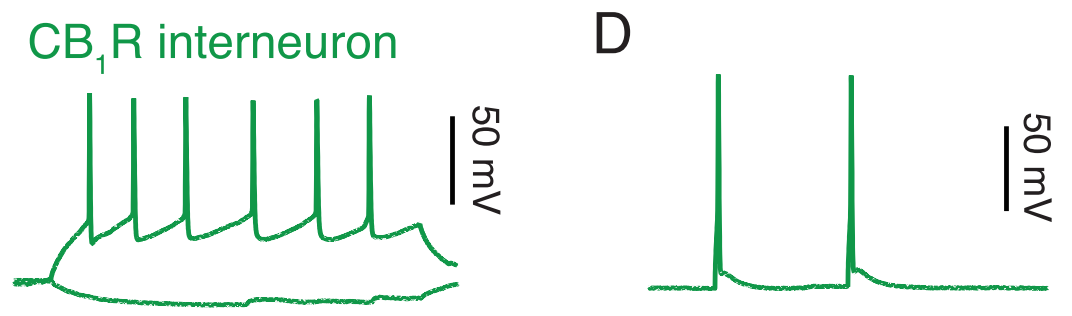

CA1 Pyramidal neuron
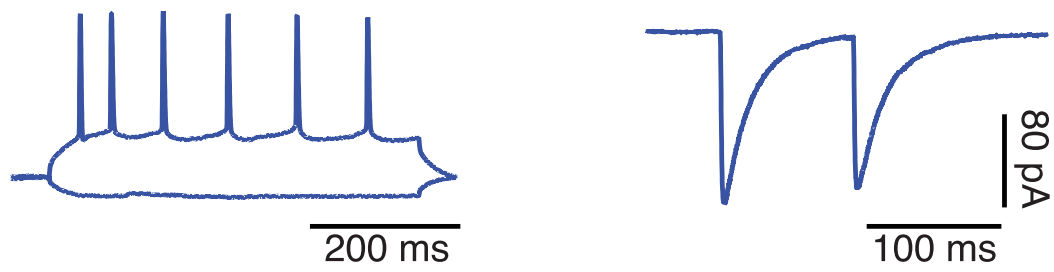

Figure 1. Properties of $C B_{1} R$-containing interneuron to $C A 1$ pyramidal neuron pairs. $A$, Reconstruction of the axonal (green) and dendritic (black) tree of a biocytin-filled CA1 basket interneuron with its soma (black) within stratum radiatum (str. rad.), close to the border with stratum pyramidale (str. pyr.). $\boldsymbol{B}$, Fluorescent image of part of the biocytin filled axon in green (top), $C_{1} R$ staining in red (middle), and merged image (bottom). All images are from the same focal plane. Note the colocalization of biocytin and $C B_{1} R$ antibody staining (white arrowheads). $C, A P$ firing in response to current injection of a $C B_{1} R$-containing interneuron (green) and a (A1 pyramidal neuron (blue). D, Example trace of APs evoked by two short current injections into the interneuron (green) and the IPSC response in a postsynaptic pyramidal neuron voltage clamped at $-70 \mathrm{mV}$ (blue; average of 50 sweeps) of the same cell pair as in $\boldsymbol{C}$.

slices containing somatosensory cortex were cut from 21- to 28-d-old FVB- $\operatorname{Tg}($ GadGFP) 45704Swn/J mice expressing EGFP under control of the mouse Gadl gene promoter (The Jackson Laboratory stock no. 003718), thereby labeling a subset of somatostatin-positive interneurons in hippocampus and neocortex (Oliva et al., 2000). Slices were maintained at $33^{\circ} \mathrm{C}$ for $\sim 30 \mathrm{~min}$ and recorded at room temperature $\left(20-22^{\circ} \mathrm{C}\right)$ in a similar solution, but now with $2 \mathrm{mM} \mathrm{CaCl}_{2}$ and $2 \mathrm{~mm}$ $\mathrm{MgCl}_{2}$.

Paired recordings of $C B_{1} R$-containing interneurons and $C A 1$ pyramidal neurons. Cellular recordings were performed from 2-6 neurons simultaneously using borosilicate glass pipettes with resistance of 3-5 $\mathrm{M} \Omega$, containing the following (in $\mathrm{mm}$ ): $70 \mathrm{~K}$ gluconate, $70 \mathrm{KCl}, 4 \mathrm{Mg}$-ATP, 4 phosphocreatine, 0.4 GTP, 0.5 EGTA and 10 HEPES (pH 7.3, KOH). In synaptically connected neurons, suprathreshold stimulation of the presynaptic interneuron evoked a $\mathrm{GABA}_{\mathrm{A}} \mathrm{R}$ mediated IPSP/IPSC in the postsynaptic CA1 pyramidal neuron. Because of the high intracellular chloride concentration, IPSCs were observed as inward currents, while IPSPs were depolarizing. Presynaptic cells were stimulated with a pair of two suprathreshold current pulses (at $\sim 10 \mathrm{~Hz}$ ). Trains were delivered with intervals of $5 \mathrm{~s}$. Recording and stimulus delivery was performed using standard electrophysiological equipment.

SSI recordings in neocortical interneurons. For experiments on SSI, EGFP expressing interneurons in somatosensory cortex were selected using an UV light source and fluorescence imaging. Extracellular and intracellular solutions were similar as for paired recordings, but to ex- clude contributions from synaptic conductances DNQX $(10 \mu \mathrm{M})$, GABAzine $(100 \mu \mathrm{M})$, and DL-AP5 $(100 \mu \mathrm{M})$ were added to the extracellular solution.

Immunohistochemistry. Some recorded interneurons were filled with biocytin $(0.5 \%)$, and slices were fixed and resliced after recording. Sections were stained using the antibodies goat-anti-streptavidin Alexa Fluor 488 conjugate $(1: 200)$ and the primary antibody rabbitanti- $\mathrm{CB}_{1} \mathrm{R}$ (diluted 1:1000 in TBS-TX) (Tsou et al., 1998). In a second step, sections were incubated with the secondary antibody Alexa Fluor 594 goat anti-rabbit IgG (1:200). Colocalization between biocytin and $\mathrm{CB}_{1} \mathrm{R}$ was determined using a Leica confocal laser-scanning microscope (Leica), the Huygens system (Scientific Volume Imaging), and the Amira isosurface module (Konrad Zuse Center for Information Technology, Berlin, Germany). Care was taken to prevent false positives, incomplete signal separation and crosstalk of dyes. Neuron reconstructions were performed under an up-right microscope (Leica) using a $40 \times$ objective with Neurolucida software (Neurolucida, MicroBrightField).

Chemicals. Of tetrahydrolipstatin (THL), RHC-80267 (Sigma-Aldrich) and OMDM-188 (Ortar et al., 2008) appropriate stock solutions were prepared in DMSO (Final concentration $<0.2 \%$ ). Slice-incubation was done in the slice chamber for at least $30 \mathrm{~min}$ before recordings started, with the same compound concentration present extracellularly. For intracellular application, intracellular equilibration was allowed for at least $20 \mathrm{~min}$ after whole-cell break-in (see Figs. 3, 4). Vehicle controls with intracellular or extracellular DMSO were interleaved with normal control experiments. Because we observed no vehicle effects, all control experiments were grouped.

Analysis. All analysis was done using custom written procedures in Igor Pro (Wavemetrics). Statistical analysis was done either using twosided Student's $t$ test (for single comparisons; $\alpha=0.05$ ), or ANOVA with post hoc Dunnett's test (for multiple comparisons; $\alpha=0.05$ ).

\section{Results}

Simultaneous whole-cell recordings were made from CA1 pyramidal neurons and interneurons located in stratum radiatum, close to the border with stratum pyramidale (Fig. 1). Cell bodies of selected interneurons had a multipolar appearance and their firing pattern upon current injection showed considerable spike-frequency adaptation (Fig. 1C). To confirm the presence of $\mathrm{CB}_{1} \mathrm{Rs}$ in the studied interneurons, we used biocytin staining and immunostaining for the $\mathrm{CB}_{1} \mathrm{R}$ in a subset of interneurons (Fig. 1A,B). All interneurons showed substantial colocalization of biocytin and $\mathrm{CB}_{1} \mathrm{R}$ immunoreactivity in axons $(n=5 / 5)$ (Fig. $1 B)$.

Inhibitory connections were activated by evoking two action potentials (APs) at $\sim 10 \mathrm{~Hz}$ in the interneuron, while recording IPSCs in the CA1 pyramidal neuron (voltage-clamped at -70 $\mathrm{mV}$ ) (Fig. $1 D$ ). In cell pairs showing an inhibitory synaptic connection we tested for the presence of DSI. Two APs were evoked in the interneuron once every $5 \mathrm{~s}(0.2 \mathrm{~Hz})$. After recording 50 control responses, the subsequent 50 responses were each pre- 
ceded by a depolarizing step ( $1 \mathrm{~s}$ to $0 \mathrm{mV}$ ) to the postsynaptic pyramidal cell (depolarization period) (Fig. 2A). For IPSCs evoked after such a depolarizing step, the IPSC amplitude was reduced to $36.0 \pm$ $2.6 \%$ of control (Fig. $2 A-C)(p=0.0001$; $n=7)$, meaning that there was $64.0 \pm$ 2.6\% DSI. Amplitude reduction was accompanied by a strong increase in synaptic failure rate (Fig. 2D) (control $30.0 \pm$ 4.6\%; depolarization $49.4 \pm 5.1 \%$; $p=$ 0.024). After the depolarization period, both IPSC amplitude and failure rate partially recovered, respectively to $76.3 \pm$ $3.1 \%$ and $37.1 \pm 5.0 \%$ (Fig. $2 A-D$ ).

The increase in failure rate during the depolarization period is in support of a presynaptic locus of suppression of inhibition. Analysis of the coefficient of variation (CV) of the IPSC amplitudes (Faber and Korn, 1991) showed that the ratio between $\mathrm{CV}^{2}$ during DSI versus $\mathrm{CV}^{2}$ under control was proportional to the relative change in synaptic amplitude (Fig. 2E) (averaged $\mathrm{CV}_{\mathrm{DSI}}^{-2} / \mathrm{CV}_{\text {control: }}^{-2} 0.38 \pm 0.03$; amplitude ratio: $0.36 \pm 0.03$ ), confirming that the modulation is presynaptic in origin. In $\mathrm{CB}_{1} \mathrm{R}$ knock-out $\left(\mathrm{CB}_{1} \mathrm{R}^{-1-}\right)$ mice, we did not observe a significant reduction in IPSC amplitude during the depolarization period, and failure rate was unaffected (supplemental Fig. S1, available at www.jneurosci.org as supplemental material). Together, these findings suggest that DSI at these unitary synapses occurs through a decreased presynaptic release probability by $\mathrm{CB}_{1} \mathrm{R}$ activation.

Activity-dependent endocannabinoid synthesis in several brain regions depends on the DAGL enzymatic activity (Melis et al., 2004; Safo and Regehr, 2005; Szabo et al., 2006). To test for the involvement of DAGLs in hippocampal DSI, we used several DAGL inhibitors. Slices were incubated with the novel potent DAGL inhibitor OMDM-188 (2 $\mu \mathrm{M}$; Ortar et al., 2008) for at least $30 \mathrm{~min}$ before commencing paired recordings in the presence of OMDM-188. Under these conditions, significant DSI was still observed (amount of DSI: $46.2 \pm 5.3 \% ; n=6 ; p=0.016$ ) (Fig. $3 A$ ), and was accompanied by an increase in failure rate (control: $51.7 \pm 1.6 \%$; depolarization: $67.7 \pm 2.2 \%$; $p=0.03$ ) (Fig. 3A). The amount of DSI did not differ significantly from control conditions $(p>0.05)$ (Fig. $3 F)$. Similarly, slices incubated and recorded with the established DAGL inhibitor RHC$80267(50-70 \mu \mathrm{M})$ also showed significant DSI $(87.5 \pm 2.4 \% ; n=$ $5 ; p=0.009)$ (Fig. $3 B$ ), and an increase in failure rate (control: $54.0 \pm 4.7 \%$; depolarization: $92.0 \pm 0.6 \% ; p=0.03$ ) (Fig. $3 B$ ).

Recently, several studies have reported opposing effects of extracellular application of the DAGL inhibitor THL on hippocampal DSI (Szabo et al., 2006; Edwards et al., 2008; Hashimotodani et al., 2008). It has been shown that intracellular application of low concentrations of THL is very effective at inhibiting DAGL activity in striatum (500 nM) (Melis et al., 2004) and cerebellum (2 $\mu \mathrm{M})$ (Safo and Regehr, 2005). Therefore, we added

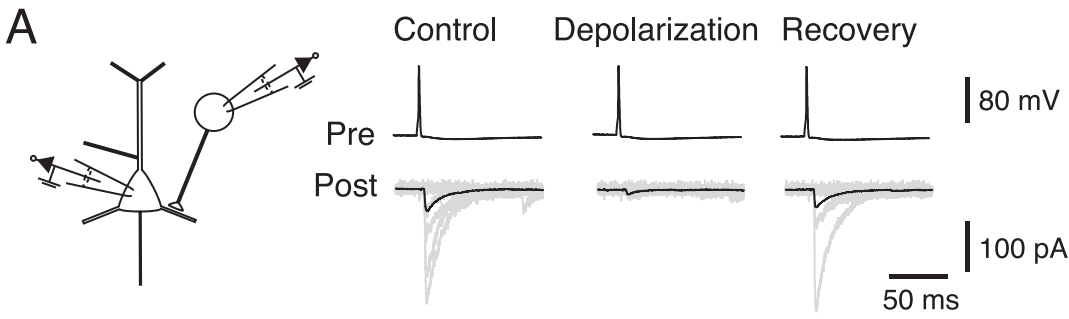

2. DSI in connected interneuron to CA1 pyramidal neurons. $\boldsymbol{A}$, Left, Recording configuration. Right, Postsynaptic IPSC Traces show 10 individual IPSC responses (gray traces) as well as the average response of 50 subsequent responses (black traces) for Failure rate during control, following depolarization and during recovery. Open circles and dotted lines represent individual experiments; closed circles and solid lines show averaged failure rate for each condition. change with DSI in coefficient of variance (CV, $y$-axis) plotted against the averaged change in IPSC amplitude ( $x$-axis). Open circles show individual experiments, closed circle shows the average.

THL $(5 \mu \mathrm{M})$ to the intracellular solution of the postsynaptic pyramidal neuron, without applying THL extracellularly. To allow intracellular diffusion of THL the depolarization protocol was started at least $20 \mathrm{~min}$ after establishing the whole-cell configuration at the pyramidal neuron. Intracellularly applied THL did not affect DSI (70.9 $\pm 1.6 \% ; n=15 ; p=0.02)$ (Fig. $3 C)$ (compared to control DSI: $p>0.05$ ) (Fig. $3 F$ ). Accordingly, failure rate significantly increased upon depolarization (control: $24.9 \pm$ 1.6\%; depolarization: $66.8 \pm 2.0 \%$; $p<0.001$ ) (Fig. $3 C$ ). Even with a $1 \mathrm{~h}$ waiting period for intracellular THL diffusion, DSI was clearly observed (Fig. 3E), suggesting that the ineffectiveness of intracellular THL was not due to incomplete intracellular diffusion. Surprisingly, when slices were incubated extracellularly with higher concentrations of THL $(10 \mu \mathrm{M})$, DSI was abolished $(17.2 \pm 2.1 \% ; n=6 ; p=0.10)$ (Fig. $3 D$ ), and failure rate was not affected by depolarization (control: $51.8 \pm 3.8 \%$; depolarization: $51.1 \pm 3.4 \% ; p=0.86$ ) (Fig. 3D). To assess whether extracellularly applied THL affected $\mathrm{CB}_{1} \mathrm{R}$ signaling downstream of the $\mathrm{CB}_{1}$ receptor, we tested the effect of THL on the suppression of IPSCs by the $\mathrm{CB}_{1} \mathrm{R}$ agonist WIN55,212-2 (2 $\left.\mu \mathrm{M}\right)$. IPSC suppression by 
A OMDM-188 (bath)
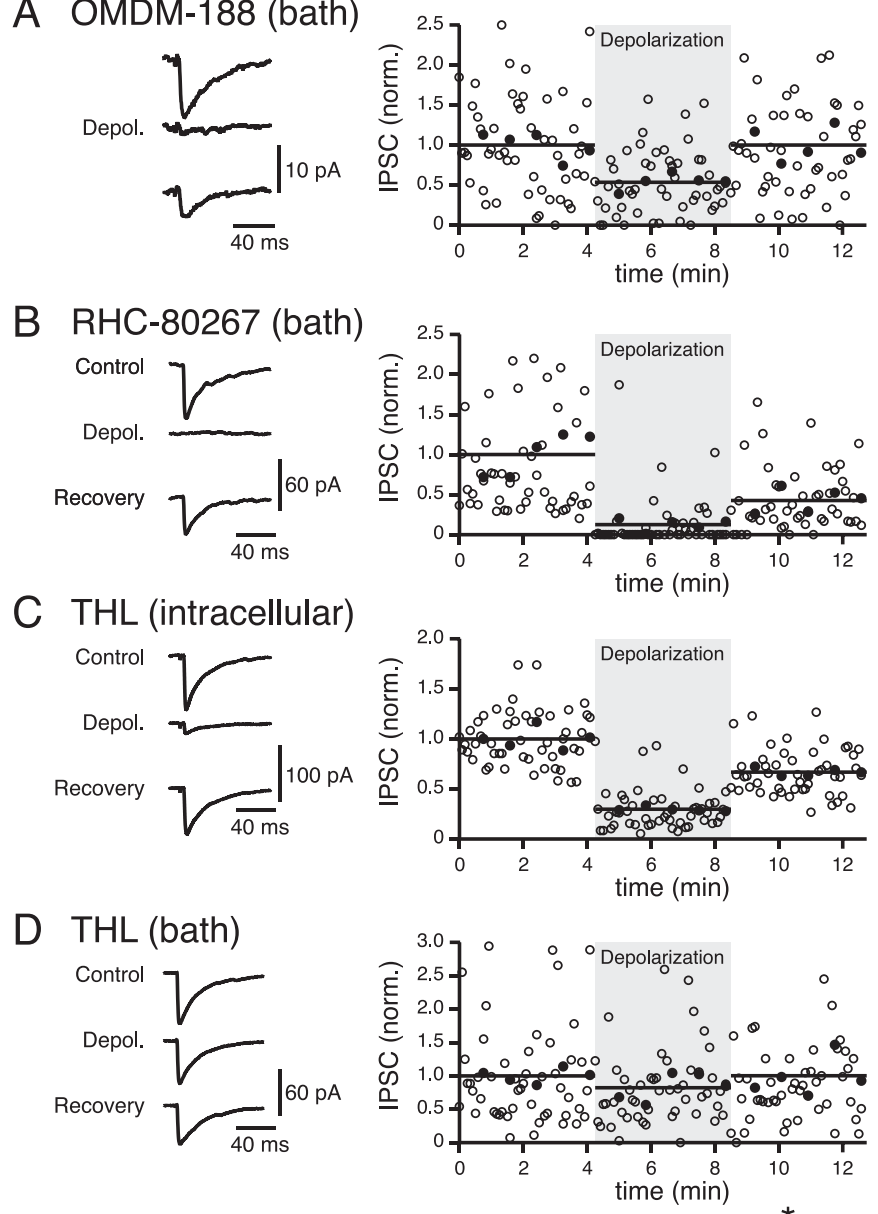

$\mathrm{E}$
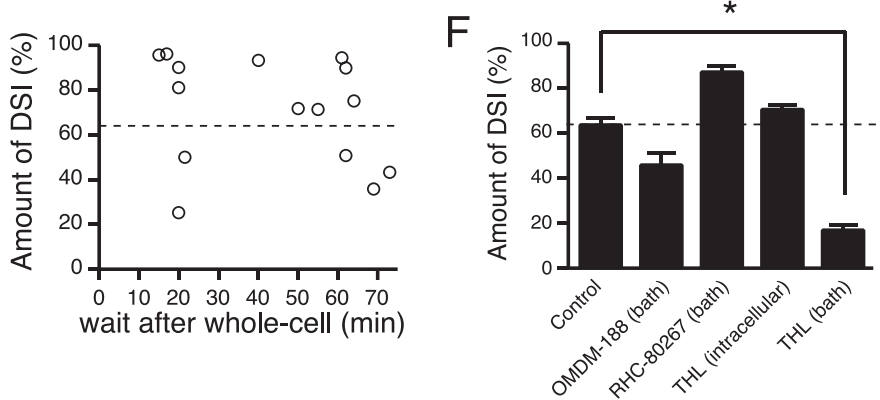

Figure 3. The effect of DAGL inhibitors on DSI. $\boldsymbol{A}$, Left, Postsynaptic IPSC responses to presynaptic stimulation in connected interneuron and CA1 pyramidal neuron incubated and recorded in the DAGL inhibitor OMDM-188 $(2 \mu \mathrm{M})$. Traces show average response of 50 subsequent responses for each condition. Middle, Average normalized IPSC amplitudes of all experiments in 0MDM-188 $(n=6)$, displayed as in Fig. 2C. Right, Failure rate during control, following depolarization and during recovery for experiments in 0MDM-188, displayed as in Fig. 2D. $\boldsymbol{B}$, Same as in $\boldsymbol{A}$, but now for experiments on slices incubated and recorded in the DAGL inhibitor RHC-80267 $(70 \mu \mathrm{m} ; n=5)$. C, Same as in $\boldsymbol{A}, \boldsymbol{B}$, but now for experiments in which THL $(5 \mu \mathrm{M})$ was added to the intracellular solution of the postsynaptic pyramidal neuron ( $n=15)$. $\boldsymbol{D}$, Same as in $\boldsymbol{A}$, $\boldsymbol{B}$, but now for experiments on slices incubated and recorded in the DAGL inhibitor THL $(10 \mu \mathrm{m} ; n=6)$. $\boldsymbol{E}$, The amount of DSI observed in experiments in which THL $(5 \mu \mathrm{M})$ was added to the intracellular solution of the postsynaptic pyramidal neuron versus the time waited after whole cell on the postsynaptic pyramidal neuron (same experiments as in $\mathbf{C}$ ). Dotted line shows the amount of DSI observed under control conditions. $\boldsymbol{F}$, Amount of DSI observed under different conditions.

WIN55,212-2 was unaffected in slices incubated in THL, showing that THL incubation did not interfere with $\mathrm{CB}_{1} \mathrm{R}$ signaling down stream of the receptor (supplemental Fig. S2, available at www. jneurosci.org as supplemental material).

These observations raise the possibility that the other treatments were not effective in inhibiting DAGLs due to suboptimal experimental conditions such as lack of cell penetration. To exclude this possibility, we used the above procedures and inhibi-
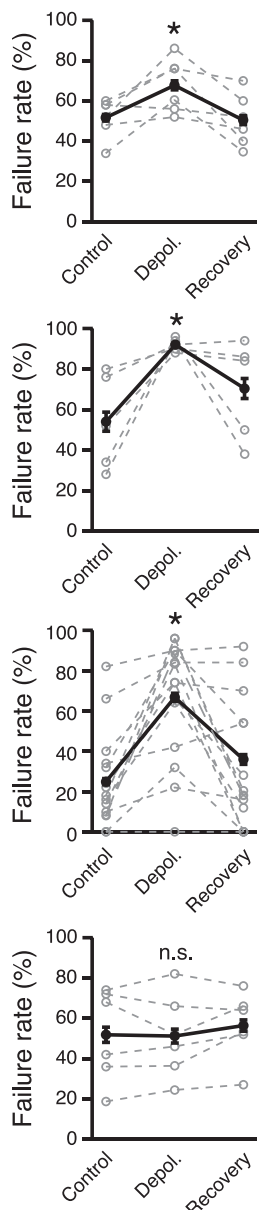

tors to block DAGLs in the well-established phenomenon of SSI of neocortical interneurons (Bacci et al., 2004). SSI is induced by repetitive AP firing of somatostatinpositive low-threshold spiking (LTS) interneurons in neocortex (Fig. 4). It is triggered by endocannabinoid-mediated activation of postsynaptic $\mathrm{CB}_{1} \mathrm{Rs}$, which in turn activate a prolonged $\mathrm{K}^{+}$conductance. Recently, it was shown that DAGL inhibition effectively abolishes SSI (Marinelli et al., 2008). We made whole-cell recordings of interneurons in mice expressing EGFP in somatostatin-positive interneurons (Oliva et al., 2000) (Fig. 4A). Upon hyperpolarizing current steps these neurons showed a clear rebound depolarization that regularly induced a rebound AP (Fig. 4A), characteristic of LTS interneurons. After recording resting membrane potential for several minutes, LTS interneurons were stimulated with 10 trains of 60 APs at 50 $\mathrm{Hz}$ each (Marinelli et al., 2008). This stimulation induced a clear, long-lasting drop in membrane potential (SSI; $\Delta V_{\mathrm{m}}$ : $-4.30 \pm 0.96 \mathrm{mV} ; n=12 ; p=0.001$ ) (Fig. $4 B)$. SSI was absent in $\mathrm{CB}_{1} \mathrm{R}^{-1-}$ mice, showing that it is mediated by $\mathrm{CB}_{1} \mathrm{Rs}$ (supplemental Fig. S3, available at www. jneurosci.org as supplemental material). All pharmacological interventions reducing DAGL-activity effectively blocked SSI (Fig. 4,C-F). When slices were incubated and recorded in OMDM-188 (2 $\mu \mathrm{M})$, SSI was absent $\left(\Delta V_{\mathrm{m}}:-0.79 \pm\right.$ $0.76 \mathrm{mV} ; n=8 ; p=0.33$ ) (Fig. $4 C$ ). Incubation with RHC-80267 (70 $\mu \mathrm{M})$ also effectively blocked SSI $\left(\Delta V_{\mathrm{m}}\right.$ : $0.63 \pm 1.34 \mathrm{mV} ; n=5 ; p=0.66$ ) (Fig. $4 D)$. Finally, intracellular application of THL $(5 \mu \mathrm{M})$ also effectively blocked SSI $\left(\Delta V_{\mathrm{m}}:-0.35 \pm 0.98 \mathrm{mV} ; n=6 ; p=\right.$ 0.74) (Fig. $4 E$ ).

Therefore, we conclude that the lack of effect of DAGL inhibitors on DSI at the interneuron to CA1 pyramidal neuron unitary connections that we studied was not due to suboptimal experimental conditions. Taken together, our data suggest that DSI at these unitary connections in hippocampus occurs without the involvement of DAGL activity.

\section{Discussion}

Biosynthetic pathways controlling endocannabinoid formation in hippocampal DSI have remained unclear. We systematically addressed involvement of DAGLs in DSI at identified unitary synaptic connections. We show that several known DAGL inhibitors do not affect DSI at these hippocampal synapses, while they abolish SSI in neocortical interneurons (Fig. 4), a process known to require DAGL activity (Marinelli et al., 2008). Our findings strongly suggest that DAGL is not involved in hippocampal DSI. 
Recently, studies have reported opposing effects of the DAGL inhibitor THL on DSI (Szabo et al., 2006; Edwards et al., 2008; Hashimotodani et al., 2008). Intracellular application of THL effectively inhibits DAGL activity in VTA, cerebellum, and neocortex (Melis et al., 2004; Safo and Regehr, 2005; Marinelli et al., 2008). We found that intracellular postsynaptic application of THL did not affect DSI. This lack of effect is unlikely to be due to experimental conditions, because intracellular THL application successfully blocked SSI, confirming a previous report by Marinelli et al. (2008). Therefore, these results confirm the findings with other DAGL inhibitors, and rule out involvement of DAGL in hippocampal DSI. This raises the question by what mechanism extracellular THL application blocks hippocampal DSI (Edwards et al., 2008; Hashimotodani et al., 2008). Hashimotodani et al. (2008) and Szabo et al. (2006) ruled out indirect effects of THL on either $\mathrm{CB}_{1} \mathrm{R}$ signaling or calcium influx necessary for DSI induction. Likewise, we show that slice incubation with THL does not affect $\mathrm{CB}_{1} \mathrm{R}$ signaling (supplemental Fig. S2, available at www.jneurosci.org as supplemental material). At present, the mechanism by which extracellular THL blocks hippocampal DSI remains elusive. Given the absence of effects of OMDM-188, RHC-80267, and intracellular THL on DSI, we argue that extracellular THL does not block DSI through inhibition of DAGL.

A recent study showed that hippocampal DSI during cholinergic receptor activation requires signaling by nitric oxide (NO) (Makara et al., 2007). This opens the possibility that under these conditions endocannabinoid synthesis takes place outside of the postsynaptic neuron, perhaps in the presynaptic terminal, and that NO is the messenger emanating from the postsynaptic neuron. If the downstream target of NO would be sensitive to THL, this might explain why extracellular application of THL blocks DSI, while postsynaptic application is ineffective. However, in the same study from Makara et al. (2007) it was shown that NO is not involved in DSI in the absence cholinergic receptor activation. In agreement with this, we find that DSI at unitary connections, in the absence of cholinergic receptor activation, is not affected by incubation with the NO-synthase inhibitor $N_{\omega}$ Nitro-L-arginine methyl ester (supplemental Fig. S4, available at www.jneurosci.org as supplemental material). Therefore, NO involvement cannot explain the discrepancy between the effects of extracellular and postsynaptic THL application in our experiments.

It has been suggested that DSI might involve a pre-formed 2-AG pool (Edwards et al., 2006), and that DAGLs are involved in filling this pool, not in the actual DSI process. However, when blocking DAGLs, we did not observe a reduction in the amount of DSI during 50 subsequent depolarizations, although this would be expected when depleting a pre-formed pool of 2-AG. Therefore, if DSI involves a pre-formed pool of 2-AG, this pool must be quite substantial and stable.
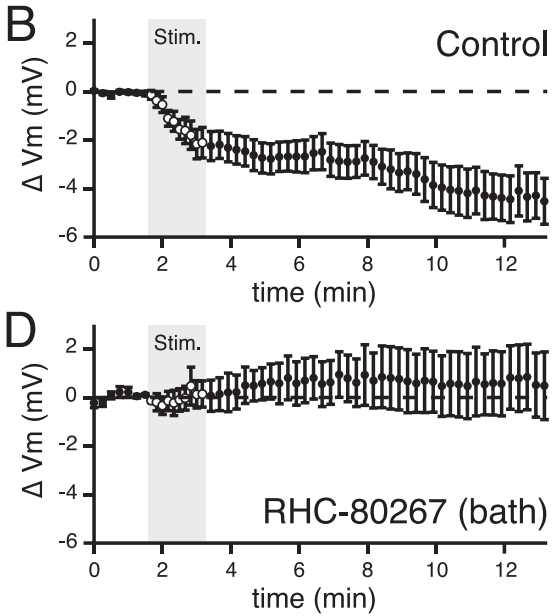

F



Figure 4. The effect of DAGL inhibitors on SSI in neocortical LTS interneurons. $A$, Left, Infrared differential interference contrast (Stim.), and following the stimulation. $\boldsymbol{C}$, Same as in $\boldsymbol{B}$, but $\boldsymbol{B}-\boldsymbol{D}$, but now for experiments in which THL $(5 \mu \mathrm{m})$ was added to the intracellular solution of the LTS interneuron $(n=6)$. $\boldsymbol{F}$, Amount of SSI observed under different conditions.

Recent studies suggested that 2-AG, and not anandamide, is the endocannabinoid mediating hippocampal DSI. This conclusion was based on the ability of inhibitors of monoacylglycerol lipase, the enzyme that breaks down 2-AG, to prolong hippocampal DSI (Makara et al., 2005; Hashimotodani et al., 2007). Furthermore, inhibition of fatty-acid amide hydrolase, the enzyme responsible for anandamide breakdown, does not affect the time course of hippocampal DSI (Kim and Alger, 2004; Makara et al., 2005; Hashimotodani et al., 2007). However, while studies of DAGL distribution in hippocampus have shown that $s n-1-D A G L-\alpha$ is perfectly positioned for production of 2-AG as retrograde messenger at excitatory synapses (Katona et al., 2006; Yoshida et al., 2006), sn-1DAGL- $\alpha$ was not found opposite symmetrical, GABAergic synapses (Katona et al., 2006). Together with our results, this suggests that $s n-1-D A G L-\alpha$ is not involved in DSI at inhibitory synapses in hippocampus. This may suggest that 2-AG is produced by different mechanisms at these synapses, involving another, hitherto unknown, synthesis pathway, such as the lysophosphatidic aciddependent route (Nakane et al., 2002). Alternatively, it may suggest that $2-\mathrm{AG}$ is not the endocannabinoid mediating DSI at unitary inhibitory synaptic connections in hippocampus.

\section{References}

Bacci A, Huguenard JR, Prince DA (2004) Long-lasting self-inhibition of neocortical interneurons mediated by endocannabinoids. Nature 431: $312-316$. 
Bisogno T, Howell F, Williams G, Minassi A, Cascio MG, Ligresti A, Matias I, Schiano-Moriello A, Paul P, Williams EJ, Gangadharan U, Hobbs C, Di Marzo V, Doherty P (2003) Cloning of the first sn 1-DAG lipases points to the spatial and temporal regulation of endocannabinoid signaling in the brain. J Cell Biol 163:463-468.

Chevaleyre V, Castillo PE (2003) Heterosynaptic LTD of hippocampal GABAergic synapses: a novel role of endocannabinoids in regulating excitability. Neuron 38:461-472.

Edwards DA, Kim J, Alger BE (2006) Multiple mechanisms of endocannabinoid response initiation in hippocampus. J Neurophysiol 95:67-75.

Edwards DA, Zhang L, Alger BE (2008) Metaplastic control of the endocannabinoid system at inhibitory synapses in hippocampus. Proc Natl Acad Sci U S A 105:8142-8147.

Faber DS, Korn H (1991) Applicability of the coefficient of variation method for analyzing synaptic plasticity. Biophys J 60:1288-1294.

Hashimotodani Y, Ohno-Shosaku T, Kano M (2007) Presynaptic monoacylglycerol lipase activity determines basal endocannabinoid tone and terminates retrograde endocannabinoid signaling in the hippocampus. J Neurosci 27:1211-1219.

Hashimotodani Y, Ohno-Shosaku T, Maejima T, Fukami K, Kano M (2008) Pharmacological evidence for the involvement of diacylglycerol lipase in depolarization-induced endocanabinoid release. Neuropharmacology 54:58-67.

Katona I, Urban GM, Wallace M, Ledent C, Jung KM, Piomelli D, Mackie K, Freund TF (2006) Molecular composition of the endocannabinoid system at glutamatergic synapses. J Neurosci 26:5628-5637.

Kim J, Alger BE (2004) Inhibition of cyclooxygenase-2 potentiates retrograde endocannabinoid effects in hippocampus. Nat Neurosci 7: 697-698.

Makara JK, Mor M, Fegley D, Szabo SI, Kathuria S, Astarita G, Duranti A, Tontini A, Tarzia G, Rivara S, Freund TF, Piomelli D (2005) Selective inhibition of 2-AG hydrolysis enhances endocannabinoid signaling in hippocampus. Nat Neurosci 8:1139-1141.

Makara JK, Katona I, Nyiri G, Nemeth B, Ledent C, Watanabe M, de Vente J, Freund TF, Hajos N (2007) Involvement of nitric oxide in depolarization-induced suppression of inhibition in hippocampal pyramidal cells during activation of cholinergic receptors. J Neurosci 27:10211-10222.

Marinelli S, Pacioni S, Bisogno T, Di Marzo V, Prince DA, Huguenard JR, Bacci A (2008) The endocannabinoid 2-arachidonoylglycerol is responsible for the slow self-inhibition in neocortical interneurons. J Neurosci 28:13532-13541.
Marsicano G, Wotjak CT, Azad SC, Bisogno T, Rammes G, Cascio MG, Hermann H, Tang J, Hofmann C, Zieglgansberger W, Di Marzo V, Lutz B (2002) The endogenous cannabinoid system controls extinction of aversive memories. Nature 418:530-534.

Melis M, Perra S, Muntoni AL, Pillolla G, Lutz B, Marsicano G, Di Marzo V, Gessa GL, Pistis M (2004) Prefrontal cortex stimulation induces 2-arachidonoyl-glycerol-mediated suppression of excitation in dopamine neurons. J Neurosci 24:10707-10715.

Nakane S, Oka S, Arai S, Waku K, Ishima Y, Tokumura A, Sugiura T (2002) 2-Arachidonoyl-sn-glycero-3-phosphate, an arachidonic acid-containing lysophosphatidic acid: occurrence and rapid enzymatic conversion to 2-arachidonoyl-sn-glycerol, a cannabinoid receptor ligand, in rat brain. Arch Biochem Biophys 402:51-58.

Oliva AA Jr, Jiang M, Lam T, Smith KL, Swann JW (2000) Novel hippocampal interneuronal subtypes identified using transgenic mice that express green fluorescent protein in GABAergic interneurons. J Neurosci 20:3354-3368.

Ortar G, Bisogno T, Ligresti A, Morera E, Nalli M, Di Marzo V (2008) Tetrahydrolipstatin analogues as modulators of endocannabinoid 2-arachidonoylglycerol metabolism. J Med Chem 51:6970-6979.

Safo PK, Regehr WG (2005) Endocannabinoids control the induction of cerebellar LTD. Neuron 48:647-659.

Sugiura T, Kondo S, Sukagawa A, Nakane S, Shinoda A, Itoh K, Yamashita A, Waku K (1995) 2-Arachidonoylglycerol: a possible endogenous cannabinoid receptor ligand in brain. Biochem Biophys Res Commun 215:89-97.

Szabo B, Urbanski MJ, Bisogno T, Di Marzo V, Mendiguren A, Baer WU, Freiman I (2006) Depolarization-induced retrograde synaptic inhibition in the mouse cerebellar cortex is mediated by 2 -arachidonoylglycerol. J Physiol 577:263-280.

Tsou K, Brown S, Sanudo-Pena MC, Mackie K, Walker JM (1998) Immunohistochemical distribution of cannabinoid $\mathrm{CB}_{1}$ receptors in the rat central nervous system. Neuroscience 83:393-411.

Wilson RI, Nicoll RA (2001) Endogenous cannabinoids mediate retrograde signalling at hippocampal synapses. Nature 410:588-592.

Yoshida T, Fukaya M, Uchigashima M, Miura E, Kamiya H, Kano M, Watanabe M (2006) Localization of diacylglycerol lipase- $\alpha$ around postsynaptic spine suggests close proximity between production site of an endocannabinoid, 2-arachidonoyl-glycerol, and presynaptic cannabinoid CB1 receptor. J Neurosci 26:4740-4751. 